\title{
Divided attention between lyrics and tunes of operatic songs: Evidence for independent processing
}

\author{
ANNE-MARIE BONNEL \\ Centre de Recherche en Neurosciences Cognitives, CNRS, Marseille, France \\ FREDERIQUE FAITA \\ Université de Bordeaux, France \\ ISABELLE PERETZ \\ Université de Montreal, Quebec, Canada \\ and \\ MIREILLE BESSON \\ Centre de Recherche en Neurosciences Cognitives, CNRS, Marseille, France
}

\begin{abstract}
Excerpts from French operatic songs were used to evaluate the extent to which language and music compete for processing resources. Do these two dimensions conflict? Are they integrated into a single percept? Or are they independent? The final word of each excerpt was either semantically congruous or incongruous relative to the prior linguistic context and was sung either in or out of key. Participants were asked to detect either the semantic or the melodic incongruity (single task) or both (dual task). We predicted a dual-task deficit if these tasks conflicted and no deficit if they were either independent or integrated. In order to distinguish between these last two outcomes, trial-by-trial contingency analyses were also computed, predicting no correlation if the tasks were conflicting or independent, a positive correlation under the assumption of integration and a negative correlation if dividing attention is impossible. Our results show similar levels of performance in single and dual tasks and no correlation between dual-task judgments, thus suggesting that semantic and melodic aspects of language are processed by independent systems. In addition, a comparison between musicians and nonmusicians shows that these conclusions are independent of musical expertise.
\end{abstract}

Vocal music is the most popular form of music, and song is a universal art form that includes two components, the lyrics and the tune. The study of the perception of songs thus entails the study of two cognitive abilities: language and music. The general question we address is the problem of the specificity of the computations involved in language processing. Although the claim for language specificity is central to some linguistic theories, such as the generative grammar (Chomsky, 1957),

This work was supported by grants from the Medical Research Council of Canada to I.P. and from the Groupement d'Intérêt Scientifique, "Science de la Cognition" (1995-1998) to M.B., E.B., and M. Habib. I.P. was supported by a fellowship from the Fonds de la Recherche en Santé du Quebec. This research was conducted while F.F. was a postdoctoral student in the Psychology Department of the University of Montreal, supported by a fellowship from the Fyssen Foundation. The authors thank Thomas O. Wickens for supplying his correct version of the Dorfman and Alf maximum-likelihood procedure, Sylvie Noel for testing most participants, and B. Repp for his helpful comments on a previous version of this paper. Correspondence concerning this article should be addressed to A. M. Bonnel, Department of Psychology, U. C. Berkeley, Berkeley, CA 94720 (e-mail: ambonnel@ socrates.berkeley.edu). other theories, in contrast, are based on the idea that language processing relies on general cognitive principles (Tomasello, 1998). By studying the relationship between lyrics and tunes in songs, we wanted to determine whether these two aspects are processed independently or whether they are integrated within a unified percept? Finding evidence for independent processing would add some arguments in favor of the specificity of language processing. In contrast, finding interactions between the processing of lyrics and tunes would argue in favor of common processing stages and, therefore, against the specificity hypothesis.

It seems quite plausible that one's hearing the melody of a well-known song can cue the text, and vice versa. Previous results have indeed shown that melodies of song are better recognized when they are heard with their original words than when they were heard with the text of another song equally familiar to the participants. Similarly, words of songs are better recognized when they are heard with the original melody than when they are heard with a different but equally familiar melody (Crowder, Serafine, \& Repp, 1990; Samson \& Zatorre, 1991; Ser- 
afine, Crowder, \& Repp, 1984; Serafine, Davidson, Crowder, \& Repp, 1986). These results have been taken as evidence for "an integration effect in memory for the melody and text of songs" (Crowder et al., 1990, p. 469).

Interestingly, however, previous research using the event related potentials (ERPs) methodology has shown a quite different pattern of results (Besson, Faita, Peretz, Bonnel, \& Requin, 1998). Recording the ERPs elicited by the final words of excerpts from French operatic songs, Besson et al. obtained evidence that semantic and melodic components of songs are processed independently. Indeed, violations of semantic and melodic expectancies were associated with different ERP effects, which did not interact. The present experiments were designed to further investigate the on-line processing of lyrics and tunes when songs are listened to, by using the same materials as Besson et al. but a different methodology. The materials comprised operatic songs, mostly from the 19th century. All excerpts were good examples of tonal Western music that generate specific expectancies. The excerpts were sung a cappella (without instruments), and each excerpt lasted between 15 and $20 \mathrm{sec}$ and ended in a monosyllabic word. This critical word was either the congruous word or another monosyllabic word semantically incongruous within the linguistic context of the libretto. Moreover, it was sung either as written in the original score or with a different pitch. Violations of expectancies on the melodic dimension were produced by singing the word out of key (i.e., out of the tonality of the musical phrase so that it sounded wrong).

When words are sung, the basic auditory stimulus that reaches the ears and activates the basilar membrane is an integration of linguistic and musical information. However, beyond this first sensory level of analysis, it remains to be determined whether semantic and melodic characteristics of songs, among other dimensions, are processed as a unified percept or as separate components. In the present study, we reasoned that to listen to songs may represent a typical case of divided attention, and we used the logic of dual tasks in order to determine whether attention to one source of information adversely affects the perception of the other. In single tasks, participants were asked to pay attention only to language and to detect incongruous words regardless of how they were sung, or to pay attention only to music and to detect out-of-key words regardless of their meaning. In dual tasks, participants were asked to divide their attention equally between language and music and to detect all types of incongruities whether semantic, melodic, or both.

\section{Do the Linguistic and Musical Tasks Share the Same Resource?}

The basic rationale of the dual-task paradigm is that the volume as well as the nature of processing resources can only be properly investigated by overloading the participant with multiple tasks and by examining the patterns of interference (Navon \& Gopher, 1979; Norman \& Bobrow, 1975). Table 1 summarizes the possible out-
Table 1

Rationale of the Dual-Task Paradigm

\begin{tabular}{lcc}
\hline & Dual-Task Outcome & Contingency \\
\hline Independence & No deficit & 0 \\
Integration & Deficit & + \\
Benefit & Deficit & 0 \\
Concurrent: sharing & Deficit & - \\
\hline
\end{tabular}

comes regarding the level of performance within a given task when performed conjointly with another. According to resource theory, insofar as two tasks demand separate resources, they will be performed together as efficiently as in isolation. Consequently, if listening to lyrics and tunes involves independent processes, dual-task performance should be as good as single-task performance. Competition can also be prevented if lyrics and tunes are somehow integrated, in which case dual-task performance would be as good, or even better than single-task performance. In contrast, competition for the same pool of resources would produce a dual-task deficit, whether participants share resources (i.e., divide their attention between lyrics and tunes on every trial), or direct their attention only toward one component on any given trial and switch between trials.

Note that the assumptions of integration and independence, although they correspond to quite different mechanisms, lead to undistinguishable predictions. Consequently, a cost-benefit analysis alone would be incomplete. One way to solve this difficulty, as is recommended by Bonnel and Prinzmetal (1998), is to combine the costbenefit approach with a trial-by-trial contingency analysis of the performances in the divided attention condition. Analysis of detection performance for semantic and melodic components within a trial allows us to distinguish between competing outcomes by revealing the attention strategy used by participants on a trial-by-trial basis when they attend to both lyrics and tunes simultaneously.

\section{Are Lyrics and Tunes Processed Independently or Jointly? Analysis of the Attention Strategy}

Positive correlation: Integration. At one extreme, linguistic and musical performances can be positively correlated (if one perceives the semantic congruity of the lyrics, one would also perceive the melodic congruity of the tunes). A positive correlation would indicate that the two components are somehow unified, either because the two components are integrated into a single percept (at the extreme, there could be only a single perceptual dimension) or because attention to a song results in the mandatory processing of all its attributes, even if they are not integrated. Results showing any type of integration would be taken as evidence that a song should be considered as an object comprising two dimensions.

Negative correlation: Switching. At the other extreme, linguistic and musical performances can be neg- 
atively correlated. Such a finding would imply that participants focus their attention on only one component on any given trial. Under the switching strategy, when the response on one task is correct, the response on the other task is likely to be wrong, and vice versa. Such an outcome would indicate that simultaneous attention to the lyrics and to the tunes is impossible, or that participants choose to avoid the burden of dividing their attention between competing sources of information.

Between these two extremes, there might be no correlation between performance on the two tasks. The existence of two distinct strategies might lead to this outcome.

No correlation: Sharing. The two tasks might well conflict, but, within trials, participants are able to share resources, thus performing part of each task on a given trial and possibly performing both tasks simultaneously. If sharing occurs within a trial, one might expect no correlation (see Sperling, 1984).

No correlation: Independence. Alternatively, there may be no competition at all, so that attention can be allocated to both components within a trial. In the present framework, this would reflect the fact that different types of resources are required to process the linguistic and musical components. Evidently, the last two strategies imply quite distinct mechanisms. Thus, the contingency analysis, although providing fine-grained information about the relation between joint processes, is incomplete. The important point, however, is that when cost-benefit and contingency analyses are combined, clear-cut predictions emerge. Table 1 illustrates the rationale of the present study, showing that the issue of same versus different resources maps closely onto the issue of divided versus focused attention. ${ }^{1}$

Within each task, detection performance was analyzed in terms of signal detection theory (SDT; Green \& Swets, 1966/1974), which provides a general model for understanding an observer's sensitivity to differences between stimuli. Sensitivity is the observer's ability to respond correctly to the stimulus presented. Response bias is the observer's tendency to preferentially use one of the two responses. The usefulness of SDT methodology is in large part due to the fact that it allows a bias-free measure of sensitivity to be estimated.

\section{Effects of Expertise}

Although results of several experiments have demonstrated differences in music processing between experts and naive listeners, the extent to which musical expertise influences perception still deserves more scrutiny. In particular, it is still not clear at which processing stage these differences occur. Rating tasks, for instance, have been widely used in music research (Cuddy \& Badertscher, 1987; Cuddy, Cohen, \& Mewhort, 1981; Cuddy \& Thompson, 1992; Krumhansl, Bharucha, \& Kessler, 1982; Palmer \& Krumhansl, 1987a, 1987b). Typically, listeners are asked to rate how well the final note completes the musical pattern or how well a chord fits into the musical context. However, such tasks do not allow one to assess which processing stage is modulated by expertise. For example, at the perceptual level, one might expect musicians to detect musical incongruities with higher accuracy than would nonmusicians. However, musical expertise may also affect response bias, if musicians adopt a more lenient criterion (i.e., are more willing than nonmusicians to give an incongruous response to the same information). SDT was used in the present experiment to further track this issue.

\section{METHOD}

\section{Single-Task Condition}

Participants. Forty-eight students (mean age, 24.7 years; range 16-34), all native French speakers from Quebec, participated in this experiment, which lasted for about one and a half hours. Sixteen were nonmusicians, among whom 3 had had 3 years of music education, but at least 8 years prior to the present study. The other 32 participants were enrolled in a full-time music education program. They had 10.5 years of formal musical training on average. Half of the musicians were singers, and half were instrumentalists. All were paid for their participation.

Materials. Two hundred different excerpts were drawn from a corpus of French operatic songs used by Besson et al. (1998). Complete musical phrases were presented so that they ended in a monosyllabic word and conveyed an impression of closure. The mean duration of the excerpts was $16 \mathrm{sec}$ (range: 13-20). Each excerpt was sung a cappella (i.e., without instruments) by a professional female singer in four different conditions. In one condition, the song matched the original score. In the three other conditions, the final word was incongruous. The incongruity was semantic, melodic, or both, relative to the preceding context. Semantic anomalies were induced by presenting the last word with a rhyming monosyllabic word of the same category (e.g., noun/noun) but with an unrelated meaning, for example boeufs (oxen) for yeux (eyes). Semantically incongruous words were sung in the original pitch in one condition, and in an anomalous pitch in another condition. Pitch anomalies were induced by replacing the original pitch of the final tone by the subtonic (a note half a tone below the main note of a key, or tonic) or a nondiatonic pitch (a note out of the scale corresponding to the tonality), so that the pitch modification created a violation of tonal expectancies. Thus, each of the 200 excerpts was sung either as written or with a semantic, melodic, or combined incongruity on the last event (see Figure 1 for an example from Meyerbeer's opera Les Huguenots and from Gounod's Faust).

The resulting 800 different excerpts were divided into four lists, so that each excerpt appeared only once in a list and was presented in each condition across lists. Moreover, each list comprised an equal number of trials of each type, so that within the experiment, each participant was presented with $50 \mathrm{ScMc}$ (semantically and melodically congruous), $50 \mathrm{SiMc}$ (semantically incongruous and melodically congruous), $50 \mathrm{ScMi}$ (semantically congruous and melodically incongruous), and $50 \mathrm{SiMi}$ (semantically and melodically incongruous) excerpts. Trials were mixed in a pseudorandom order so that no more than three musical phrases of the same type were presented consecutively. The four lists were recorded on digital audiotapes, with a time interval of $4 \mathrm{sec}$ mean duration between two musical excerpts. The excerpts were presented via a Sony DATrecorder through two loudspeakers placed in front of the participant.

Procedure. The participants in each group (nonmusicians, instrumentalists, and singers) were assigned randomly, and in equal number, to one of the four lists. Half of them performed the linguistic task, and the other half performed the musical task. In the 


\section{LES HUGUENOTS}

Meyerbeer (1791-1864)
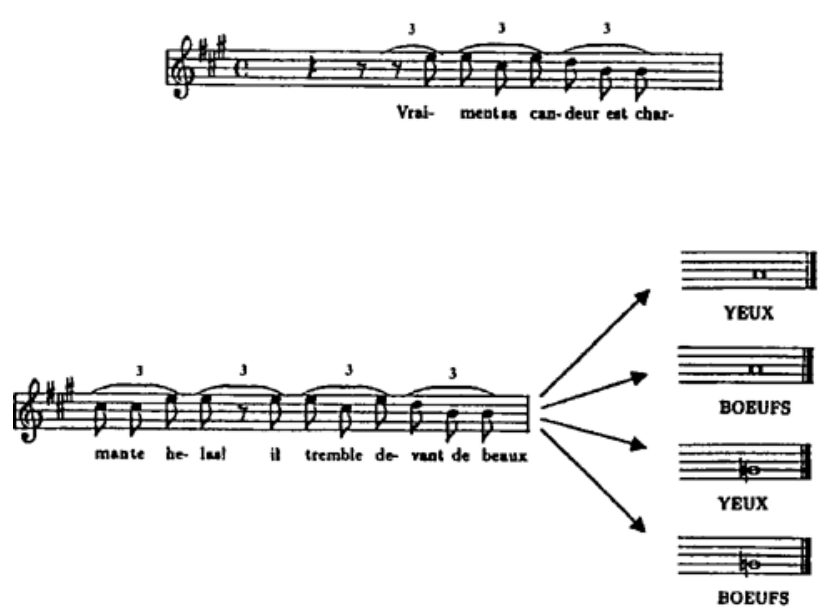

FAUST

Gounod (1818-1893)
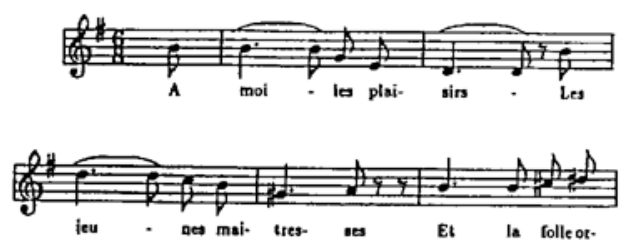

Figure 1. Example of the materials used in the experiment. Note that, in French, the final incongruous word boeufs (oxen) rhymes with the expected completion yeux (eyes). The final note is either in key (lines 1 and 2) or out of key (lines 3 and 4 ).

linguistic task, the participants were asked to pay attention only to the language in order to detect semantic incongruities while ignoring the pitch variations. They had to judge on a 6-point scale whether or not the final word was incongruous in the sentence context, with $1=$ absolutely certain congruous and $6=$ absolutely certain incongruous. Conversely, in the music task, the participants were asked to pay attention only to the music in order to detect melodic incongruities while ignoring the semantic content of the sentences. They had to judge on the same 6-point scale whether or not the final pitch sounded incongruous (out of key). Finally, all participants were asked to judge on the 6-point scale whether or not the excerpt sounded familiar. The familiarity judgment was considered a secondary task and was performed after the main incongruity detection task. The participants responded during the time interval between two excerpts. At the beginning of the session, eight excerpts from operatic songs, which were not repeated in the experiment, were presented in each of the four possible conditions in order to familiarize the participants with the voice of the singer, the duration of the excerpts, and the task at hand.

\section{Dual-Task Condition}

Participants. Twenty-four students (mean age, 23.9 years; range, 18-40) who did not participate in the preceding experiment were selected according to the same criteria. There were 8 nonmusicians, 8 singers, and 8 instrumentalists. The musicians had an average of 11.6 years of formal training. All were paid for their participation.

Materials and Procedure. The materials and procedure were identical to those used in the single-task condition, except that the participants were instructed to pay attention both to the semantic aspects of language and to the melodic aspects of music. For each excerpt, the participants were required to judge whether the final word and its pitch were congruent or incongruent with respect to the linguistic and musical contexts. They responded on two separate confidence scales located one next to the other: One scale was used for the semantic judgment and the other for the melodic judgment. The order of responding (language or music first) was counterbalanced within each group. Familiarity of each excerpt was rated after the detection task.

\section{Data Analysis}

SDT analysis. Data from each task (detection of semantic and melodic incongruities, respectively) were summarized by using standard procedures for estimating sensitivity and response bias from individual receiver-operating characteristic (ROC) curves (see Macmillan \& Creelman, 1991).

Sensitivity. For each detection task, congruous and incongruous responses and confidence judgments were combined to obtain six response categories, from high-confidence incongruous to highconfidence congruous. The five boundaries between adjacent pairs of these categories yielded five points on a ROC curve from which optimal estimates of the parameters of each ROC, as well as their variance, were computed with the use of an iterative maximumlikelihood ratio estimate, using the procedures yielded by Dorfman and Alf (1969). From each individual ROC curve, sensitivity was assessed by using the nonparametric index $d_{a}$, which is a monotonic function of the area under the ROC curve (see Macmillan \& Creelman, 1991). This index is free from the assumption of equal variances of the underlying distributions (i.e., noise alone and signal + noise). In other words, the estimate of sensitivity is not biased when the distribution generated by congruous events does not have the same variance as the distribution generated by incongruous ones.

Statistical comparisons between parameters were carried out with the use of $z$ tests, as recommended by Dorfman and Alf (1969). Since the parameter estimation procedure gives not only the estimated value of each ROC parameter but also the variance of this estimate, $z$ values were used to test whether the parameter $d_{a}$ associated with one condition was statistically different from an estimate of the same parameter from another condition, using the standard error of the estimate averaged across participants.

Response bias. One of the possible measures of response bias is the location of the criterion, which provides a single bias score indicating point $B$ on the observer's rating scale, at which he or she 
uses both responses equally. Because all ROCs did not show unit slopes, which indicates that in some conditions the two underlying distributions did not have equal variance, a nonparametric index was computed. The $B$ index (McNicol, 1972) indicates the rating scale category at which $P($ Hits $)+P(\mathrm{FA})=1$. Thus, in the present experiment, with a 6-point scale and five categories, $B=3.5$ meant that the participant used incongruous and congruous responses equally. However, the limitation of $B$ is that it does not provide a bias measure for each criterion, so that, if an experimental condition has led the participant not to move all his or her criteria up or down the $x$-axis, but rather to space them more widely apart or closer together, $B$ will not detect these changes. Thus, direct observation of all the points on the ROCs was also used.

Contingency analysis. Finally, contingency analyses were conducted on dual-task data. For each component (semantic and melodic), congruous and incongruous responses were scored as correct or incorrect, and the results on each trial were tabulated in a $2 \times 2$ table; rows indicated the accuracy for one task (language), and columns indicated the accuracy for the other (music). The $\varphi$ coefficient or fourfold point correlation (Hays, 1994) was used to measure the contingency observed in each $2 \times 2$ table and tested perceptual independence (Ashby \& Townsend, 1986). This index ranges from -1 (total negative dependence) to +1 (total positive dependence) and is equal to zero when the two components are perceived independently (i.e., are statistically independent). For each participant, the $\varphi$ coefficient was based on 200 observations. Statistical signif icance was computed from these values by using the corresponding $\varphi\left(\varphi^{2}=N \varphi^{2}\right)$.

\section{RESULTS}

\section{Sensitivity}

Table 2 presents SDT analysis of ROC data and reports the mean and standard error of the estimates computed for each participant. The slopes of the average ROCs are generally quite close to 1 , a result confirmed by statistical analysis. The departure from unity observed in the overall detection of semantic incongruities (average slope $=1.285)$ is not significant $(z=0.90$, n.s. $)$. Neither is the opposite trend observed in the detection of melodic incongruities, whether overall performance is considered (average slope $=0.948, z=0.20$, n.s.) or whether the group of musicians who performed the dual task is considered (average slope $=0.913, z=0.30$, n.s.). Although the difference between the slopes observed in the detection of semantic and melodic incongruities is quite large (average slope $=1.285$ vs. 0.948 ), this effect does not reach significance, either in the single task $(z=$ 1.34$, n.s. $)$ or in the dual task $(z=1.03$, n.s.).
In a preliminary analysis, we compared sensitivity $\left(d_{a}\right.$ index) between the two groups of professional musicians. As can be seen in Table 2, these two groups were quite similar. Statistical analysis of their respective performance in single tasks confirmed that the difference between instrumentalists and singers was not significant, either for the detection of semantic incongruities $(z=$ 0.357, n.s.) or for the detection of melodic incongruities $(z=0.151$, n.s.). Similarly, the difference between these two groups in the dual tasks was not significant, either for the detection of semantic incongruities $(z=0.604$, n.s.) or for the detection of melodic incongruities $(z=$ 0.774 , n.s.). Consequently, instrumentalists and singers were treated as a single group in further analysis. The complete SDT analysis in the form of empirical ROCs is presented in Figure 2 for musicians and nonmusicians. In order to increase the stability of the estimated parameters for model fitting, responses were pooled across participants before the iterative maximum-likelihood procedure was used to obtain optimal estimates of the parameters of each ROC (Macmillan \& Kaplan, 1985). Figure 2 illustrates the homogeneity of the data for the detection of semantic incongruities across groups and conditions. The mean $d^{\prime}$ differences across groups are quite small $(0.239$ in the single task, and 0.014 in the dual task). The differences across conditions are also small ( 0.044 for the nonmusicians, and 0.209 for the musicians). In this task, errors most likely result from the fact that incongruous words rhymed with the expected congruous completions; both words could therefore be mistaken. ROC curves observed for the detection of melodic incongruities are somewhat more differentiated, mainly because musicians performed better than did nonmusicians. In this task, errors most likely resulted from the use of subtonics as incongruous endings. Although the subtonic favors a feeling of incompletion, it may not always be considered as incongruous. This is corroborated by Besson and Faita's (1995) findings that subtonic endings generate more errors $(57.8 \%)$ than do nondiatonic incongruities (32.6\%).

\section{Cost-Benefit Analysis}

Most important for the present purpose, we found no deficit in dual-task, as compared with single-task, performance. Statistical analysis of sensitivity estimates within

Table 2

Estimated Receiver-Operating Characteristic Parameters as a Function of Attention, Task, and Musical Expertise

\begin{tabular}{|c|c|c|c|c|c|c|c|c|c|c|c|c|c|c|c|c|}
\hline \multirow[b]{4}{*}{ Expertise } & \multicolumn{8}{|c|}{ Single-Task Condition } & \multicolumn{8}{|c|}{ Dual-Task Condition } \\
\hline & \multicolumn{4}{|c|}{ Semantic Judgment } & \multicolumn{4}{|c|}{ Melodic Judgment } & \multicolumn{4}{|c|}{ Semantic Judgment } & \multicolumn{4}{|c|}{ Melodic Judgment } \\
\hline & \multicolumn{2}{|c|}{ Slope } & \multicolumn{2}{|c|}{$d_{a}$} & \multicolumn{2}{|c|}{ Slope } & \multicolumn{2}{|c|}{$d_{a}$} & \multicolumn{2}{|c|}{ Slope } & \multicolumn{2}{|c|}{$d_{a}$} & \multicolumn{2}{|c|}{ Slope } & \multicolumn{2}{|c|}{$d_{a}$} \\
\hline & $M$ & $S D$ & $M$ & $S D$ & $M$ & $S D$ & $M$ & $S D$ & $M$ & $S D$ & $M$ & $S D$ & $M$ & $S D$ & $M$ & $S D$ \\
\hline Ionmusician & 31 & 200 & 968 & 0.192 & 0.890 & 0.142 & 132 & 0.172 & 1.344 & 0.339 & 1.924 & 0.191 & 1.058 & 0.201 & 1.603 & 0.185 \\
\hline Instrumer & 1.355 & 0.291 & 1.696 & & 0.914 & 0.272 & 2.512 & & 1.103 & & 1.877 & 0.194 & 0.985 & 0.297 & 2.282 & 0.251 \\
\hline Singers & 1.253 & 0.314 & 1.762 & & 0.999 & 0.347 & 2.476 & & & & 1.999 & 10 & 0.841 & 0. & 2.076 & 0.282 \\
\hline Musicians & 1.304 & 0.302 & 1.729 & 0.185 & 0.956 & 0.309 & 2.494 & & 1.221 & 0.321 & 1.938 & 0.202 & 0.913 & 0.289 & 2.179 & 0.266 \\
\hline Mean & 1.308 & 0.305 & 1.809 & 0.188 & 0.934 & 0.254 & 2.075 & 0.217 & 1.262 & 0.327 & 1.933 & 0.198 & 0.961 & 0.260 & 1.987 & 0.239 \\
\hline
\end{tabular}




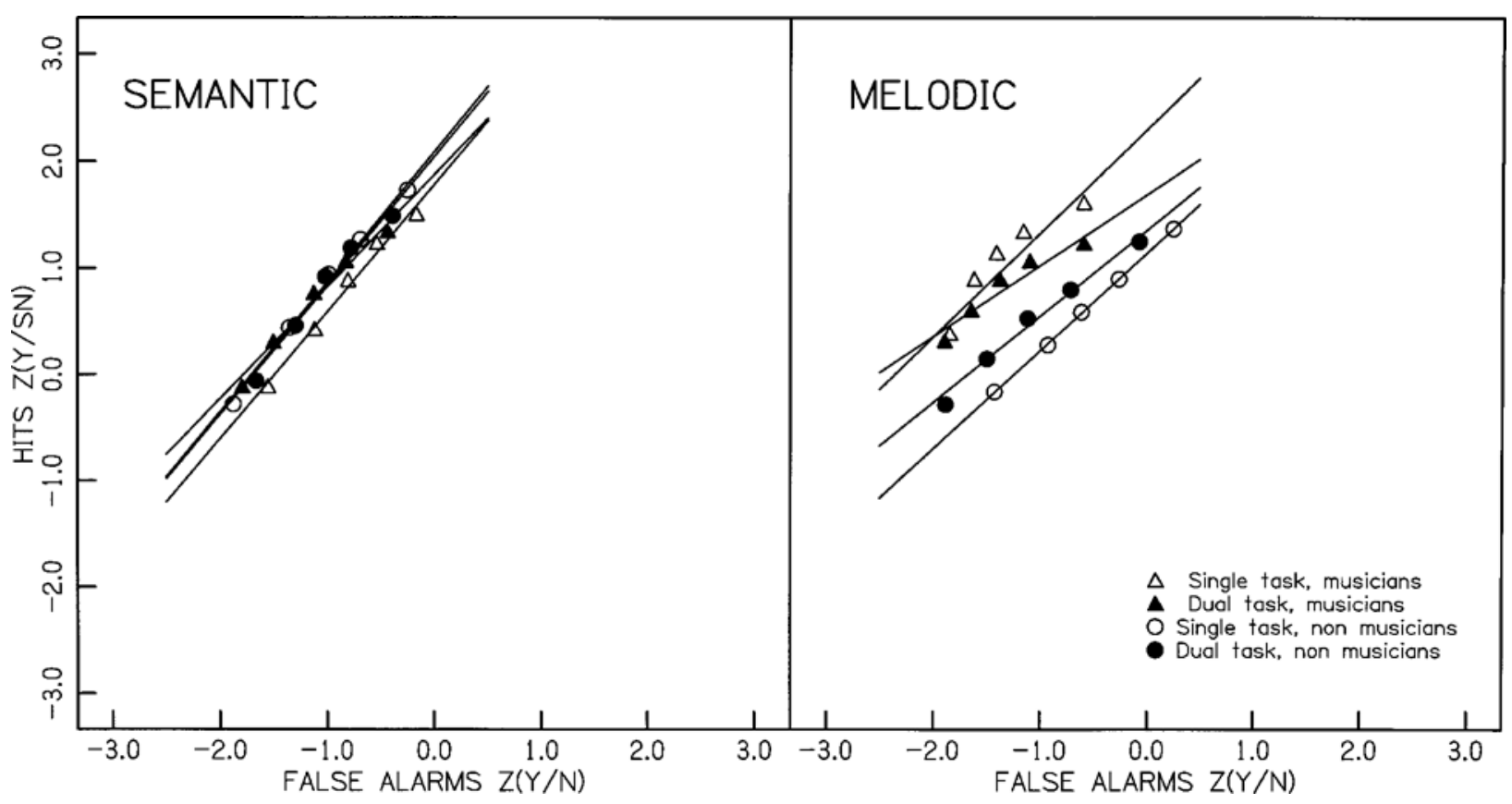

Figure 2. Receiver-operating characteristic curves for the pooled data. The axes are standard scores. Single tasks are represented by empty symbols, dual tasks by closed symbols. Musicians are represented by triangles and nonmusicians by circles.

each group (nonmusicians and musicians) confirmed that single- and dual-task performances for the detection of semantic incongruities did not differ significantly, either among the nonmusicians $(z=0.230$, n.s. $)$ or among the musicians $(z=1.082$, n.s. $)$. Similarly, the detection of melodic incongruities among musicians was not significantly impaired in the dual task $(z=1.250$, n.s.), whereas it was slightly improved among nonmusicians.

The absence of a dual-task deficit could reflect "data limits," the task's being too easy. This interpretation is not likely, however, since all performances were precisely in the range generally considered to be "resource limited" (Norman \& Bobrow, 1975). The estimated $d_{a}$ index ranges between 1.24 and 2.49, and is thus located between floor and ceiling.

In a further investigation, we conducted an analysis on two separate subsets of data. We analyzed detection per- formance on one dimension (semantic or melodic) as a function of whether or not the stimulus was congruous or incongruous on the other dimension (semantic or melodic). In the first analysis, the task (semantic or melodic) was accompanied by a congruous component. In the second, it was accompanied by an incongruous component. The aim was to test whether the presence of an incongruity in the irrelevant dimension would modify performance on the relevant dimension. These separate analyses were conducted (1) for the single tasks (in which the other dimension was to be ignored), and (2) for the dual tasks (in which the other dimension was to be attended to). The results are presented in Table 3 , in terms of the $d_{a}$ index averaged across participants. The advantage of the congruous stimuli that is apparent in most conditions is actually quite small, and none of the comparisons reached significance. In the single task, an advantage was found

Table 3

Signal Detection Theory Analysis of Sensitivity, $d_{a}$ Index, Contingent on the Nature of the Other Component as a Function of Attention, Task, and Musical Expertise

\begin{tabular}{|c|c|c|c|c|c|c|c|c|c|c|c|c|c|c|c|c|}
\hline \multirow[b]{4}{*}{ Expertise } & \multicolumn{8}{|c|}{ Single-Task Condition } & \multicolumn{8}{|c|}{ Dual-Task Condition } \\
\hline & \multicolumn{4}{|c|}{ Semantic Judgment } & \multicolumn{4}{|c|}{ Melodic Judgment } & \multicolumn{4}{|c|}{ Semantic Judgment } & \multicolumn{4}{|c|}{ Melodic Judgment } \\
\hline & \multicolumn{2}{|c|}{$\mathrm{Mc}$} & \multicolumn{2}{|c|}{$\mathrm{Mi}$} & \multicolumn{2}{|c|}{$\mathrm{Sc}$} & \multicolumn{2}{|c|}{$\mathrm{Si}$} & \multicolumn{2}{|c|}{$\mathrm{Mc}$} & \multicolumn{2}{|c|}{$\mathrm{Mi}$} & \multicolumn{2}{|c|}{$\mathrm{Sc}$} & \multicolumn{2}{|c|}{$\mathrm{Si}$} \\
\hline & $M$ & $S D$ & $M$ & $S D$ & $M$ & $S D$ & $M$ & $S D$ & $M$ & $S D$ & $M$ & $S D$ & $M$ & $S D$ & $M$ & $S D$ \\
\hline Nonmusicians & 2.060 & 0.295 & 2.020 & 0.299 & 1.383 & 0.260 & 1.385 & 0.254 & 2.027 & 0.309 & 1.841 & 0.267 & 1.760 & 0.276 & 1.515 & 0.265 \\
\hline Instrumentalists & 1.804 & 0.269 & 1.615 & 0.266 & 2.477 & 0.349 & 2.495 & & 2.012 & 0.304 & 1.617 & 0.285 & 2.326 & 0.460 & 2.425 & 0.444 \\
\hline Singers & 1.790 & 0.291 & 1.731 & 0.275 & 2.670 & 0.393 & 2.481 & & 2.259 & 0.352 & 1.901 & 0.286 & 2.345 & 0.422 & 1.984 & 0.365 \\
\hline Musicians & 1.797 & 0.280 & 1.673 & 0.270 & 2.574 & 0.371 & 2.488 & 0.387 & 2.135 & 0.328 & 1.759 & 0.285 & 2.335 & 0.441 & 2.204 & 0.404 \\
\hline Mean & 1.885 & 0.285 & 1.789 & 0.280 & 2.177 & 0.334 & 2.120 & 0.343 & 2.099 & 0.322 & 1.786 & 0.279 & 2.144 & 0.386 & 1.975 & 0.358 \\
\hline
\end{tabular}

Note- $-\mathrm{Mc}=$ melodically congruous (in tune), $\mathrm{Mi}=$ melodically incongruous (out of tune), $\mathrm{Sc}=$ semantically congruous, $\mathrm{Si}=$ semantically incongruous. 

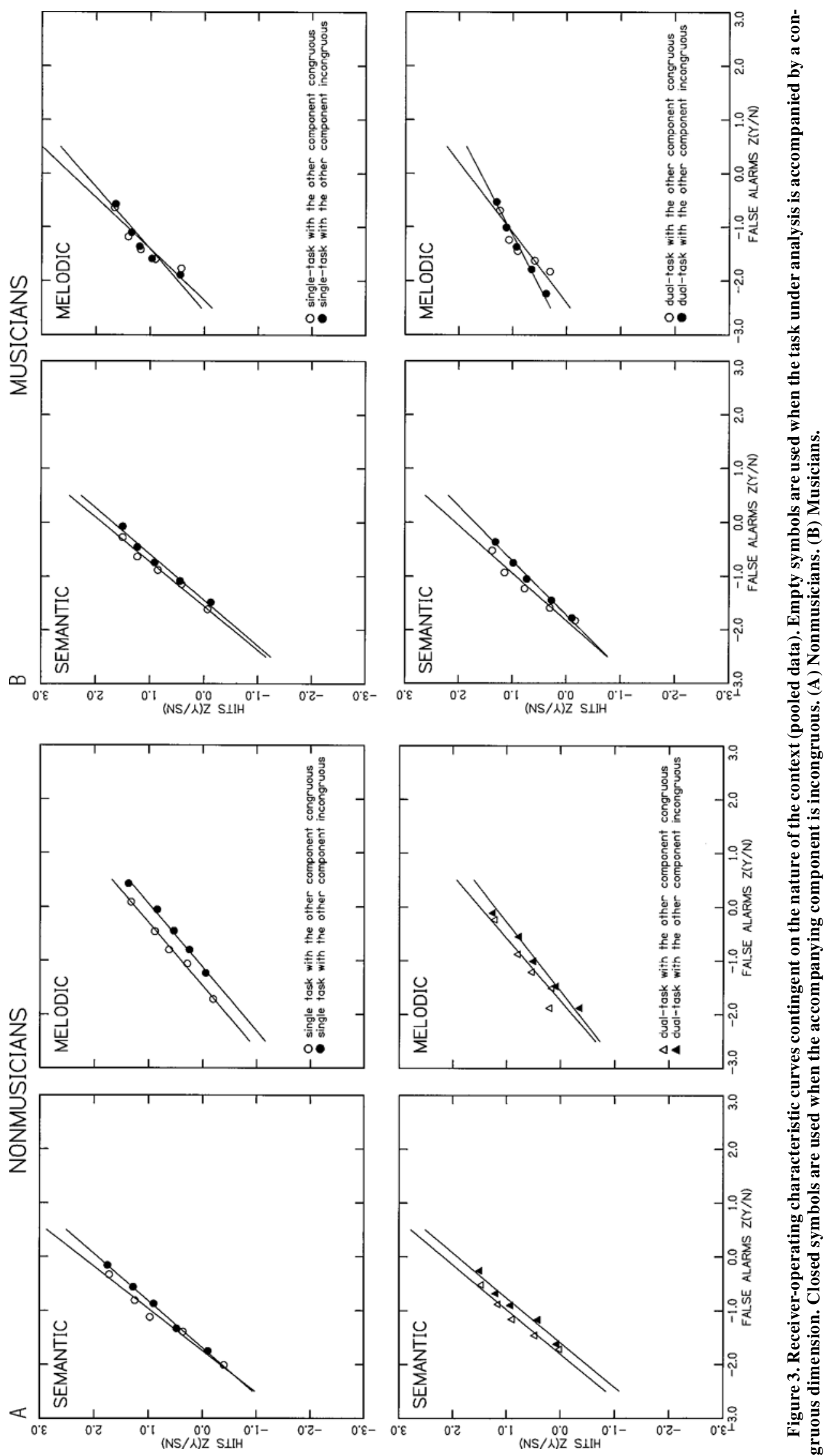
in the detection of semantic incongruities for the nonmusicians, with a difference in the average $d_{a}$ index of $0.04(z=0.067$, n.s.). In the group of musicians, a small advantage of 0.12 is found for the detection of semantic incongruities $(z=0.225$, n.s. $)$ and of 0.08 for the detection of melodic incongruities $(z=0.113$, n.s.). In the dual task, among the nonmusicians these differences were 0.19 for the detection of semantic incongruities $(z=$ 0.323 , n.s.) and $0.24(z=0.453$, n.s.) for the detection of melodic incongruities. In the group of musicians, these differences were $0.38(z=0.613$, n.s. $)$ and $0.13(z=$ 0.155 , n.s.), respectively.

The results of the SDT analysis of ROC data are illustrated in Figure 3 (Figures 3A and 3B for the nonmusicians and for the musicians, respectively). Once again, responses were pooled across participants in each group before the iterative procedure was used. Although a small shift in the same direction is apparent in Figure 3A, performance was not significantly affected by the nature of the accompanying component, which gives additional support to the independence assumption.

\section{Effect of Expertise}

As is shown in Table 2, expertise strongly influenced the detection of melodic incongruities. Musicians performed significantly better than did nonmusicians in the single task $(z=6.142, p<.001)$ and the dual task $(z=$ $2.554, p<.01)$. In contrast, and not surprisingly, the detection of semantic incongruities was not influenced by musical expertise (single task, $z=1.268$, n.s.; dual task, $z=0.074$, n.s.). Note that in single tasks nonmusicians were more accurate at detecting semantic than melodic incongruities $(z=4.03, p<.001)$, whereas the reverse was true for musicians $(z=3.608, p<.001)$. Although similar trends were observed in dual tasks, they did not reach significance (for nonmusicians, $z=1.707$, n.s.; for musicians, $z=1.029$, n.s.). As is shown in Table 2, the lowest performance was found for nonmusicians when they were to detect melodic incongruities, whereas the highest performance was found in the same task for musicians.

Two rapid conclusions can be drawn from the analysis of accuracy. First, these data are inconsistent with the assumption of competition between language and music. The processes involved in the detection of semantic and melodic incongruities did not compete with each other, and this held for both musicians and nonmusicians. Second, musical expertise clearly improved the detection of melodic incongruities.

Table 4

Signal Detection Theory Analysis of Response Bias, $B$ Index, as a Function of Attention, Task, and Musical Expertise

\begin{tabular}{lccccc}
\hline & \multicolumn{2}{c}{ Single-Task Condition } & & \multicolumn{2}{c}{ Dual-Task Condition } \\
\cline { 2 - 3 } \cline { 5 - 6 } \multicolumn{1}{c}{ Expertise } & Semantic & Melodic & & Semantic & Melodic \\
Judgment & Judgment & & Judgment & Judgment \\
\hline Nonmusicians & 3.44 & 2.82 & & 3.20 & 3.97 \\
Musicians & 2.92 & 3.39 & & 3.65 & 3.92 \\
\hline
\end{tabular}

\section{Response Bias}

Individual values of $B$ were computed and averaged for each group of participants. Table 4 summarizes the results of this SDT analysis of response strategy averaged across participants. Overall, the response criterion is located at 3.43, which indicates that the participants were not biased toward either of the two responses. This was further confirmed by statistical analyses showing that the overall response criterion $B$ was not significantly different from $3.5(t=0.702$, n.s. $)$. In addition, neither musical expertise $[F(2,93)=0.19, p=.83]$ nor the nature of the task [detection of semantic vs. melodic incongruities; $F(1,94)=1.96, p=.16$ ] influenced criterion location. However, a one-way analysis of variance (ANOVA) showed that the criterion was significantly higher in dual tasks $(B=3.68)$ than in single tasks $(B=3.14)[F(1,94)=$ $9.17, p=.003]$. Participants thus tended to be more cautious in giving an incongruent response in the dual task.

Finally, we also analyzed response strategy on one dimension (semantic or melodic) as a function of whether or not the stimulus was congruent or incongruent on the other dimension. In this more detailed analysis, instead of using a single bias score as was done in the preceding analysis, we compared the location of each of the five criteria (from the 6-point rating scale). This comparison was achieved by comparing response criteria on the corresponding ROCs along the horizontal dimension (i.e., the probability of false alarms for each criterion). Figures 3A and $3 \mathrm{~B}$ illustrate once again that the location of the response criteria remained identical in both contexts. Therefore, response strategy on one dimension was unaffected by the nature of the component on the other dimension, and this result was independent of musical expertise.

\section{Contingencies}

Table 5 describes the detailed results of the contingency analysis performed in the dual-task condition. The three leftmost columns present the percentage of correct responses observed for each component (semantic and melodic) and for both. Then, for comparison, the value predicted under the assumption of independence, $P(\mathrm{~S}) \times$ $P(\mathrm{M}) \times 100$ is presented, followed by the values of the $\chi^{2}$ coefficient and the corresponding $\varphi$. For each of the 24 participants, the $\varphi$ index was based on 200 observations, thus, the critical $\varphi$ values are 0.138 and 0.182 for $p<.05$ and $p<.01$, respectively. Of the 24 correlation coefficients computed for each participant in the dual task, none was significantly negative, and only one was significantly positive. Within each group of participants, the mean value was close to zero. This pattern of results is clearly inconsistent with both the switching and the integration assumptions, but in line with the independence assumption, the perception of each components' being in no way contingent on the perception of the other. This pattern of results is quite surprising given the wellknown tendency to find at least some positive contingencies due to uncontrolled external factors known to affect both performances conjointly, such as occasional 
Table 5

Trial-By-Trial Analysis of Individual Data in the Dual Task

\begin{tabular}{|c|c|c|c|c|c|c|}
\hline Subject & Semantic & Melodic & Both & Independence & $\varphi$ & $\chi^{2}$ \\
\hline \multicolumn{7}{|c|}{ Nonmusicians } \\
\hline 1 & 83.0 & 81.5 & 69.5 & 68.0 & 0.13 & 3.22 \\
\hline 2 & 81.0 & 75.0 & 63.0 & 61.0 & 0.13 & 3.48 \\
\hline 7 & 85.0 & 88.5 & 75.5 & 75.0 & 0.04 & 0.34 \\
\hline 8 & 79.5 & 73.5 & 59.0 & 58.0 & 0.03 & 0.20 \\
\hline 13 & 86.0 & 87.0 & 75.0 & 75.0 & 0.01 & 0.00 \\
\hline 14 & 88.0 & 72.5 & 65.5 & 64.0 & 0.12 & 2.75 \\
\hline 19 & 80.5 & 75.0 & 62.5 & 60.0 & 0.12 & 3.07 \\
\hline 20 & 84.5 & 75.0 & 65.0 & 63.0 & 0.10 & 2.15 \\
\hline Mean & 83.4 & 78.5 & 66.9 & 65.5 & 0.08 & \\
\hline \multicolumn{7}{|c|}{ Instrumentalists } \\
\hline 3 & 61.5 & 92.5 & 56.5 & 57.0 & -0.03 & 0.18 \\
\hline 4 & 76.5 & 79.0 & 62.5 & 60.0 & 0.12 & 2.83 \\
\hline 9 & 83.5 & 91.5 & 76.0 & 76.0 & -0.04 & 0.30 \\
\hline 10 & 89.5 & 88.5 & 79.0 & 79.0 & -0.02 & 0.00 \\
\hline 15 & 86.5 & 90.5 & 77.0 & 78.0 & -0.13 & 3.28 \\
\hline 16 & 87.5 & 90.5 & 79.5 & 79.0 & 0.03 & 0.21 \\
\hline 21 & 80.0 & 81.0 & 64.0 & 65.0 & -0.05 & 0.52 \\
\hline 22 & 84.0 & 83.0 & 69.0 & 70.0 & -0.05 & 0.55 \\
\hline Mean & 81.5 & 87.0 & 70.0 & 70.5 & -0.02 & \\
\hline \multicolumn{7}{|c|}{ Singers } \\
\hline 5 & 73.0 & 83.5 & 65.5 & 61.0 & 0.28 & $15.2 * * *$ \\
\hline 6 & 74.0 & 86.5 & 65.0 & 64.0 & 0.07 & 0.87 \\
\hline 11 & 88.5 & 87.0 & 76.0 & 77.0 & -0.13 & 3.29 \\
\hline 12 & 86.0 & 89.0 & 76.0 & 76.0 & -0.09 & 1.66 \\
\hline 17 & 85.0 & 84.0 & 71.0 & 71.0 & -0.03 & 0.19 \\
\hline 18 & 87.0 & 80.0 & 71.0 & 70.0 & 0.10 & 2.17 \\
\hline 23 & 87.0 & 86.0 & 75.5 & 75.0 & 0.06 & 0.68 \\
\hline 24 & 90.0 & 92.0 & 83.0 & 83.0 & 0.02 & 0.13 \\
\hline Mean & 83.8 & 86.0 & 72.9 & 72.1 & 0.003 & \\
\hline
\end{tabular}

lapses of attention (e.g., sneezing). Furthermore, the ease of detection undoubtedly varied across musical excerpts. Although some violations were clear-cut, others were less obvious. Finally, the strength of expectations may also vary from one excerpt to the other.

Therefore, we further examined the preceding analysis for each condition separately (SiMi, ScMc, SiMc, and $\mathrm{ScMi}$ ) in order to test for the possibility that some positive contingencies were hidden by some negative contingencies. Although positive contingencies could be expected when both components were of the same nature (SiMi and ScMc conditions), negative contingencies could be expected when the components were of a different nature (ScMi and $\mathrm{SiMc})$. In this more detailed analysis, presented in Table 6 , the coefficient was based on 50 observations for each participant. The critical $\varphi$ values are thus \pm 0.277 and \pm 0.364 (for $p<.05$ and $p<$ .01 , respectively). Results show that we did not find more negative contingencies when the components were different than when they were similar; none of the 24 indices were significantly negative. Moreover, contingency was not systematically higher when the components were of the same nature. Although there was a tendency toward this direction when both the semantic and the melodic components were congruent, only 7 out of the 24 coefficients were significantly positive. On the whole, the small number of significantly positive contingencies and the small value of the mean for each condition supports quite convincingly the independence assumption. Note an exception, however: For unknown reasons, 1 participant in the group of singers (Subject 5) showed a positive correlation in three out of the four conditions.

\section{Familiarity}

Correlation between familiarity and accuracy could not be computed because the excerpts were in most cases unfamiliar to the participants. Among the nonmusicians and the instrumentalists, familiarity was quite low, with $2 \%$ to $6 \%$ of the excerpts being judged as familiar. Not surprisingly, familiarity was somewhat higher for singers ( $15 \%$ of the excerpts were judged to be familiar).

\section{DISCUSSION}

How are lyrics and tunes perceived when people listen to songs? Results of the two analyses we performed to answer this question (dual-task outcome and contingency analyses) converge to suggest that speech and music are processed independently. When asked to detect semantic and melodic incongruities at the end of 
Table 6

Correlation Analysis in the Dual Task, $\varphi$ Index, and Analysis of Individual Data Contingent on the Nature of Both Components

\begin{tabular}{|c|c|c|c|c|}
\hline Subject & $\mathrm{SiHi}$ & $\mathrm{ScHc}$ & $\mathrm{SiHc}$ & $\mathrm{ScHi}$ \\
\hline \multicolumn{5}{|c|}{ Nonmusicians } \\
\hline 1 & 0.245 & $0.582 * * *$ & -0.128 & -0.219 \\
\hline 2 & -0.182 & $0.447 * *$ & 0.147 & 0.058 \\
\hline 7 & $0.345^{*}$ & 0.119 & 0.072 & -0.221 \\
\hline 8 & $0.406^{* *}$ & 0.069 & -0.167 & 0.033 \\
\hline 13 & -0.109 & -0.167 & -0.145 & 0.206 \\
\hline 14 & 0.200 & $0.285^{*}$ & -0.042 & 0.113 \\
\hline 19 & 0.037 & 0.118 & 0.163 & 0.118 \\
\hline 20 & 0.169 & -0.090 & -0.121 & 0.236 \\
\hline Mean & 0.139 & 0.170 & -0.028 & 0.040 \\
\hline \multicolumn{5}{|c|}{ Instrumentalists } \\
\hline 3 & 0.212 & 0.148 & undefined & -0.173 \\
\hline 4 & 0.022 & $0.585^{* * *} *$ & -0.016 & -0.005 \\
\hline 9 & -0.263 & $0.414^{* * * *}$ & undefined & 0.068 \\
\hline 10 & -0.013 & -0.053 & -0.036 & -0.063 \\
\hline 15 & -0.187 & -0.111 & -0.128 & -0.068 \\
\hline 16 & 0.174 & -0.076 & -0.042 & 0.054 \\
\hline 21 & -0.177 & -0.029 & 0.049 & -0.109 \\
\hline 22 & -0.123 & -0.156 & 0.059 & -0.080 \\
\hline Mean & 0.044 & 0.090 & -0.020 & 0.081 \\
\hline \multicolumn{5}{|c|}{ Singers } \\
\hline 5 & $0.487 * * *$ & $0.387 * *$ & -0.118 & $0.341^{*}$ \\
\hline 6 & -0.119 & $0.697 * * *$ & -0.146 & 0.086 \\
\hline 11 & -0.102 & 0.201 & -0.196 & -0.118 \\
\hline 12 & -0.156 & 0.188 & -0.068 & -0.207 \\
\hline 17 & 0.239 & -0.042 & -0.085 & -0.242 \\
\hline 18 & -0.040 & -0.036 & -0.110 & $0.386 * *$ \\
\hline 23 & 0.134 & -0.042 & -0.189 & 0.218 \\
\hline 24 & -0.074 & -0.060 & 0.218 & undefined \\
\hline Mean & 0.046 & 0.1625 & -0.090 & 0.067 \\
\hline
\end{tabular}

${ }^{*} p<.05, * * p<.01, * * * p<.001$

opera excerpts, participants, regardless of their musical expertise, did not show any dual-task deficit, and there was no correlation between performances within a trial.

We also considered the possibility that the participants were unable or unwilling to share their attention between the semantic and the melodic components of songs. In this case, they may have switched their attention between components, either from one trial to the next or very rapidly within a trial. This was not the case, however. In the absence of a negative correlation, the possibility of trial-by-trial switching can be discarded. Moreover, in the absence of any dual-task deficit, the possibility of very fast switching within a trial can also be discarded. Indeed, the pattern of results showed that the participants were able to divide their attention between lyrics and tunes, and that the processes involved in performing the semantic and melodic detection tasks did not compete for the same pool of resources. The finding that the two components were independently perceived, or that perception of one component remained unaffected by perception of the other, argues against the assumption that the semantic and melodic components were integrated within a unified percept. From this perspective, a song does not seem to form a single object having two di- mensions, but rather seems to be composed of two separate objects that have one dimension each.

Interestingly, the present results stand in marked contrast with most results in the literature on divided attention. Typically, performance is reduced when more than one task has to be performed and/or when more than one attribute needs to be attended to. Dual-task deficit is thus a prevalent finding, especially when the two components belong to the same sensory modality. Although to our knowledge no results have yet been reported in the auditory domain, evidence of switching has been found in the visual domain when observers must monitor multiple locations in order to process information about conjunctions of features (Bonnel \& Prinzmetal, 1998; Shaw, 1982; Sperling \& Melchner, 1978a, 1978b). Furthermore, evidence of sharing has been found when observers must monitor multiple locations in order to process unidimensional attributes (Baron, 1973; Bonnel \& Miller, 1994; Bonnel, Possamai, \& Schmitt, 1987; Bonnel, Stein, \& Bertucci, 1992; Hugues, 1984; J. Miller \& Bonnel, 1994; Pylyshyn \& Storm, 1988). A sharing strategy has also been found when observers divide their attention between auditory and visual signals (Bonnel \& Hafter, 1998). The finding of independence is thus an atypical finding that may result either from the task we used (detection) or from the specific characteristics of the auditory stimuli. These two possibilities are considered in turn below.

Several studies have demonstrated that task requirements can be a crucial factor influencing the cost of divided attention. Typically, dual-task performance remains unaffected in detection tasks but is significantly impaired in identification tasks (Bonnel et al., 1992). The finding of no dual-task deficit might therefore reflect the fact that the participants were asked to detect incongruous events at the semantic or melodic level, but not to identify the nature of the violations. At the stimulus level, it is important to assess whether the two components that composed the stimulus were compatible or not. Results by Bonnel and Prinzmetal (1998) showed that when participants were asked to divide their attention between two dimensions of an object, the shape and the color of a letter, identification of the color (blue or green) and shape (F or T) was performed without deficit. Moreover, the strong positive correlation between the two performances indicated that when the participants knew the shape of the letter, they also knew its color. However, in this study, the color (surface attribute) filled in the shape (boundary attribute) of the letter, so that the two dimensions were physically integrated within the object. The same seems to be true for songs. Insofar as the words are sung a cappella, they form part of the stimulus that defines the tune. Nevertheless, our results are in the opposite direction.

\section{On-Line Perception of Songs Versus Memory for Songs}

It is interesting to compare the present data with those reported by Serafine et al. (1984) and Serafine et al. 
(1986) in experiments designed to investigate memory for the melody of songs. Participants listened to excerpts of folksongs that they were later asked to discriminate from new songs. When the songs were new, only the words (the lyrics), only the melody (the tunes), or both the melody and the words (the lyrics and the tunes) were new. Interestingly, old melodies were also paired with old words from another song (mismatch condition). Results showed that recognition performance was higher when both the words and the melodies were old than when one component was new. Most important, songs in the mismatch condition were not recognized as accurately as were the old songs. These results were taken as evidence for an integration of the melody and text of songs in memory. Moreover, further results showed that the semantic component was not necessary to facilitate recall of the melody of songs, since similar results had been found when nonsense words were used instead of real words. Thus, the authors argue, nonsemantic aspects of the text, such as phonology or prosody, might be responsible for the integration effects. However, although such factors may have played a role in our experiment as well, the results clearly showed independence rather than integration of the words and the melodies. One might consider that, although lyrics and tunes are perceived independently, as reflected by on-line measures of processing (Besson et al., 1998), they are later integrated in memory to form a unified representation of songs (Serafine et al., 1984; Serafine et al., 1986). Under this assumption, we should find evidence both for independent perceptual processing of lyrics and tunes and for later integration in memory, when both perception and memory are investigated within a single experiment. Finally, note that several differences exist between previous studies and the present one. In particular, results supporting the integration hypothesis were obtained using simple folksongs, whereas our material was more complex. In a study of memory for ballads, Wallace (1994) has shown that the facilitatory effect of music on recall was suppressed when the material was more complex (i.e., the musical phrases had an elaborate melodic contour, a complex rhythm, and large pitch interval sizes). Thus, differences in musical materials might also possibly account for the apparent contradictory results across studies.

\section{Neuropsychological Evidence}

Overall, our results are in line with results in the neuropsychological literature, showing that at least some aspects of music and language processing are implemented in different areas in the brain. For instance, brain disease can produce severe mental retardation, including language impairment, and spare the music faculty, as is seen in musical idiot savants (L. K. Miller, 1989). Similarly, brain injuries in adults can spare musical abilities in the presence of profound disturbances in language (Assal, 1973; Basso \& Capitani, 1985; Luria, Tsvetkova, \& Futer,
1965; Signoret, Van Eeckout, Poncet, \& Castaigne, 1987). Conversely, brain damage can produce selective breakdown of musical abilities (Griffith et al., 1997; Peretz, Belleville, \& Fontaine, 1997; Peretz et al., 1994). Thus, the current evidence suggests the existence of specific neural networks devoted to some aspects of music and language processing.

The neuropsychological evidence for separability between music and speech does, however, rely on betweendomains comparisons, by assessing music and speech perception in isolation from one another. For example, in four different studies (Griffith et al., 1997; Peretz, 1996; Peretz et al., 1997; Peretz et al., 1994), all patients as well as normal controls were able to recognize the lyrics from well-known songs, whereas all patients failed to recognize the corresponding melodic line, due to unilateral (Griffith et al., 1997) or bilateral (Peretz et al., 1997; Peretz et al., 1994) brain damage. In contrast, Samson and Zatorre (1991), in using the task and materials of Serafine et al. (1984; Serafine et al., 1986), were able to demonstrate that patients with excision of portions of their temporal lobes on either the left or the right side behaved as if they could not access the melody without having access to the text, and vice versa. Similar findings have been reported in normal adults (Crowder et al., 1990; Serafine et al., 1984; Serafine et al., 1986) and in preschool children (Morrongiello \& Roes, 1990).

It should be pointed out, however, that all these data are derived from the use of the same experimental design. It is thus plausible that the integration effects are task bound or materials specific. Given unfamiliar, interfering materials among the response choices, participants may form integrated representations of melody and text in memory in order to facilitate recognition. Such a strategy might not be efficient for the recognition of well-known songs, which are typically built around a few melodic lines, each of which can carry different lyrics. Consequently, the encoding of melody and text independently in normal situations might be more parsimonious and efficient. Indeed, Goodglass and Calderon (1997) showed opposite laterality effects (and by inference, opposite cerebral hemispheric superiority) in the recall of musical and linguistic content of the same sung digits. This dissociation, in terms of cerebral hemispheres, is more consistent with the present data than are the ones obtained with the memory recognition paradigm for unfamiliar songs.

\section{Effect of Expertise}

The results show a clear effect of musical training. Musicians from both singing and instrumental traditions were more accurate in detecting melodic incongruities in the opera excerpts than were nonmusicians. This advantage, which manifested itself in sensitivity measures, cannot be reduced to a simple effect of response confidence, since response bias was similar across groups and conditions. 
To conclude, comparison of the results obtained in the single and dual tasks argues in favor of an independent processing of lyrics and tunes when people listen to operatic songs. This interpretation is further supported by the results of the contingency analysis, showing no correlation between the detection of semantic and melodic anomalies in the dual task. Taken together, these results are in line with those reported by Besson et al. (1998) based on the analysis of changes in brain electrical activity. Both series of experiments support the idea that at least the semantic aspects of language processing require computations that are specific to language. Of course, although we demonstrated that the semantic component of language and the melodic component of music are processed independently, it is for future work to show whether similar conclusions would hold when the syntactic or phonological components of language are compared with the harmonic or rhythmic components of music.

\section{REFERENCES}

Ashby, G., \& Townsend, J. (1986). Varieties of perceptual independence. Psychological Review, 93, 154-179.

Assal, G. (1973). Aphasie de Wernicke chez un pianiste. Revue Neurologique, 29, 251-255.

Baron, J. (1973). Perceptual dependence: Evidence for an internal threshold. Perception \& Psychophysics, 13, 527-533.

Basso, A., \& Capitani, E. (1985). Spared musical abilities in a conductor with global aphasia and ideomotor apraxia. Journal of Neurology, Neurosurgery, \& Psychiatry, 48, 407-412.

Besson, M., \& FAITA, F. (1995). An event-related potential (E.R.P.) study of musical expectancy: Comparison of musicians with nonmusicians. Journal of Experimental Psychology: Human Perception \& Performance, 21, 1278-1296.

Besson, M., Faita, F., Peretz, I., Bonnel, A. M., \& Requin, J. (1998). Singing in the brain: Independence of lyrics and tunes. Psychological Science, 9, 494-498.

Bonnel, A. M., \& Hafter, E. R. (1998). Divided attention between simultaneous auditory and visual signals. Perception \& Psychophysics, 60, 179-190.

Bonnel, A. M., \& Miller, J. (1994). Attentional effects on concurrent psychophysical discriminations: Investigations of a sample-size model. Perception \& Psychophysics, 55, 162-179.

Bonnel, A. M., Possamai, C., \& Schmitt, M. (1987). Early modulations of visual input: A study of attentional strategies. Quarterly Journal of Experimental Psychology, 39A, 757-776.

Bonnel, A. M., \& Prinzmetal, W. (1998). Dividing attention between the color and the shape of objects. Perception \& Psychophysics, 60 , 113-124.

Bonnel, A. M., Stein, J. F., \& Bertucci, P. (1992). Does attention modulate the perception of luminance changes? Quarterly Journal of Experimental Psychology, 44A, 601-626.

Chomsky, N. (1957). Syntactic structures. The Hague: Mouton.

Crowder, R. G., Serafine, M. L., \& Repp, B. (1990). Physical interaction and association by contiguity in memory for the words and melodies of songs. Memory \& Cognition, 18, 469-476.

Cuddy, L. L., \& BAdertscher, B. (1987). Recovery of the tonal hierarchy: Some comparisons across age and levels of musical experience. Perception \& Psychophysics, 41, 609-620.

Cuddy, L. L., Cohen, A. J., \& Mewhort, D. J. K. (1981). Perception of structure in short melodic sequences. Journal of Experimental Psychology: Human Perception \& Performance, 7, 869-883.

Cuddy, L. L., \& Thompson, W. F. (1992). Asymmetry of perceived key movements in choral sequences: Converging evidence from a probetone analysis. Psychological Research, 54, 51-59.

Dorfman, D. D., \& Alf, E., JR. (1969). Maximum likelihood estimation of parameters of signal detection theory and determination of confidence intervals: Rating method data. Journal of Mathematical Psychology, 6, 487-496.

Goodglass, H., \& CAlderon, M. (1997). Parallel processing of verbal and musical stimuli in right and left hemispheres. Neuropsychologia, 15, 397-407.

Green, D. M., \& Swets, J. A. (1966). Signal detection theory and psychophysics. New York: Wiley. (Reprinted 1974)

Griffith, T., Rees, A., Witton, C., Cross, P., Shakir, R., \& Green, G. (1997). Spatial and temporal auditory processing deficits following right hemisphere infarction: A psychophysical study. Brain, 120, 785-794.

HAY S, W. L. (1994). Statistics (5th ed.). London: Holt, Rinehart \& Winston.

Hugues, H. C. (1984). Effects of flash luminance and positional expectancies on visual response latency. Perception \& Psychophysics, 36, 177-184.

Krumhansl, C. L., Bharucha, J. J., \& Kessler, E. J. (1982). Perceived harmonic structure of chords in three related musical keys. Journal of Experimental Psychology: Human Perception \& Performance, 8, 21-36.

Luria, A. R. Tsvetkova, L. S., \& Futer, J. (1965). Aphasia in a composer. Journal of Neurological Science, 2, 288-292.

Macmillan, N. A., \& Creelman, C. D. (1991). Detection theory: A user's guide. New York: Cambridge University Press.

Macmillan, N. A., \& Kaplan, H. L. (1985). Detection analysis of group data: Estimating sensitivity from average hit and false-alarm rates. Psychological Bulletin, 98, 185-199.

MCNicoL, D. (1972). A primer of signal detection theory. George Allen \& Unwin.

Miller,J., \& Bonnel, A. M. (1994). Switching or sharing in dual-task line-length discrimination? Perception \& Psychophysics, 56, 431446.

Miller, L. K. (1989). Musical savants: Exceptional skill in the mentally retarded. Hillsdale, NJ: Erlbaum.

Morrongiello, B. A., \& Roes, C. L. (1990). Children's memory for new songs: Integration or independent storage of words and tunes? Journal of Experimental Child Psychology, 50, 25-38.

NAVON, D., \& Gopher, D. (1979). On the economy of the human processing system. Psychological Review, 86, 214-255.

Norman, D. A., \& Bobrow, D. G. (1975). On data-limited and resourcelimited processes. Cognitive Psychology, 7, 44-64.

Palmer, C., \& Krumhansl, C. L. (1987a). Independent temporal and pitch structures in determination of musical phrases. Journal of Experimental Psychology: Human Perception \& Performance, 13, 116126.

Palmer, C., \& Krumhansl, C. L. (1987b). Pitch and temporal contributions to musical phrase perception: Effects of harmony, performance timing, and familiarity. Perception \& Psychophysics, 41, 505518.

Peretz, I. (1996). Can we lose memory for music? The case of music agnosia in a non-musician. Journal of Cognitive Neuroscience, $\mathbf{8}$, 481-496.

Peretz, I., Belleville, S., \& Fontaine, F. S. (1997). Dissociations entre musique et langage après atteinte cerebrale: Un nouveau cas d'amusie sans aphasie. Revue Canadienne de Psychologie Expérimentale, 51, 354-367.

Peretz, I., Kolinsky, R., Tramo, M., Labrecque, R, Hublet, C., Demeurisse, G., \& Belleville, S. (1994). Functional dissociations following bilateral lesions of auditory cortex. Brain, 117, 1283-1301.

Pylyshyn, Z W., \& Storm, R. W. (1988). Tracking multiple independent targets: Evidence for a parallel tracking mechanism. Spatial Vision, 3, 179-197.

SAmSON, S., \& Zatorre, R. J. (1991). Recognition memory for text and melody of songs after unilateral temporal lobe lesion: Evidence for dual-encoding. Journal of Experimental Psychology: Learning, Memory, \& Cognition, 17, 793-804.

Serafine, M. L., Crowder, R. G., \& Repp, B. H. (1984). Integration of melody and text in memory for songs. Cognition, 16, 285-303.

Serafine, M. L., Davidson, J., Crowder, R. G., \& Repp, B. H. (1986). On the nature of melody-text integration in memory for songs. Journal of Memory \& Language, 25, 123-135.

SHAW, M. L. (1982). Attending to multiple sources of information: The 
integration of information in decision making. Cognitive Psychology, 14, 353-409.

Signoret, J. L., Van Eeckout, P., Poncet, M., \& Castaigne, P. (1987). Aphasie sans amusie chez un organiste aveugle. Revue Neurologique, 143, 172-181.

Sperling, G. (1984). A unified theory of attention and signal detection. In R. Parasuraman \& D. R. Davies (Eds.), Varieties of attention (pp. 103-181). New York: Academic Press.

Sperling, G., \& Melchner, M. (1978a). The attention operating characteristic: Examples from visual search. Science, 202, 315-318.

SPerling, G., \& Melchner, M. (1978b). Visual search, visual attention, and the attention operating characteristic. In J. Requin (Ed.), Attention and performance VII (pp. 675-686). Hillsdale, NJ: Erlbaum.

Tomasello, M. (1998). The new psychology of language: Cognitive and functional approaches to language structure. Mahwah, NJ: Erlbaum.
Wallace, W. T. (1994). Effect of melody on recall of text. Journal of Experimental Psychology: Learning, Memory, \& Cognition, 20, 1471-1485.

\section{NOTE}

1. It is worth noting that cost-benefit and contingency analyses are two separate tests. Consequently, although we might expect these tests to be empirically correlated, they are logically unrelated.

(Manuscript received March 14, 2000; revision accepted for publication December 31, 2000.)

\section{NATO Advanced Research Workshop The Nature of Time: Geometry, Physics and Perception}

On May 21-24, 2002, the NATO Advanced Research Workshop on The Nature of Time: Geometry, Physics and Perception will be held in Tatranska Lomnica, The Slovak Republic. For further information, please see the Web site http://www.astro.sk/ msaniga/nato_arw/obj.html or contact

Dr. Metod Saniga

Astronomical Institute

Slovack Academy of Sciences

Tatranska Lomnica

Slovak Republic

e-mail: msaniga@ta3.sk 